\title{
Management of urachal cancer: A review by the Canadian Urological Association and Genitourinary Medical Oncologists of Canada
}

Zineb Hamilou, MD ${ }^{1}$; Scott North, MD, FRCPC ${ }^{2}$; Christina Canil, MD, FRCPC ${ }^{3}$; Lori Wood, MD, FRCPC ${ }^{4}$; Sebastien Hotte, MD, MSc, FRCPC ${ }^{5}$; Srikala Sridhar, MD, MSc, FRCPC $^{6}$; Denis Soulières, MD, FRCPC ${ }^{1}$; Mathieu Latour, $\mathrm{MD}^{7}$; Daniel Taussky, $\mathrm{MD}^{8}$; Wassim Kassouf, MD, CM, FRCSC ${ }^{9}$; Normand Blais, MD, MSc, FRCPC ${ }^{1}$

${ }^{1}$ Centre Hospitalier de l’Université de Montréal, Division of Oncology, Montreal, QC, Canada; ${ }^{2}$ University of Alberta Cross Cancer Institute, Division of Medical Oncology Edmonton, AB, Canada; ${ }^{3}$ University of Ottawa, Division of Medical Oncology, Ottawa, ON, Canada; ${ }^{4}$ Dalhousie University, Division of Medical Oncology, Halifax, NS, Canada; ${ }^{5}$ McMaster University, Division of Medical Oncology, Hamilton, ON, Canada; ${ }^{6}$ Princess Margaret Cancer Center, Division of Medical Oncology, Toronto, ON, Canada; ${ }^{7}$ Centre Hospitalier de l’Université de Montréal, Département de Pathologie et Biologie Cellulaire, Montreal, QC, Canada; ${ }^{8}$ Centre Hospitalier de l’Université de Montréal Département de Radiologie, Radio-oncologie et Médecine Nucléaire, Montreal, QC, Canada; ${ }^{9}$ McGill University Health Center, Division of Urology, Montreal, QC, Canada

Cite as: Can Urol Assoc J 2019 July 23; Epub ahead of print. http://dx.doi.org/10.5489/cuaj.5946

Published online July 23, 2019

$* * *$

\section{Introduction}

The urachus is a fibrous remnant of the allantois. After birth, it remains throughout life as the medial umbilical ligament running from the apex of the bladder to the umbilicus but without any further physiological role. First described by Begg in 1931, urachal cancer is a rare pathology representing less than $1 \%$ of bladder cancers. ${ }^{1}$ Usually located on the serous side of the bladder apex, these tumors are classically silent because of their extravesical and extraperitoneal location. Therefore, most patients present with local invasion or metastatic disease and thus are detected at a higher stage.

Based on its rarity, there have been no large prospective randomized trial in urachal cancer and there are no evidence-based guidelines on the management of this disease. The existing evidence in the medical literature is derived from case series.

In this article, led by the Genitourinary Medical Oncologists of Canada (GUMOC), we aim to review the literature of this rare disease in order to establish a Canadian consensus statement on the management of urachal cancer. To our knowledge, there is no other consensus statement available. 


\section{Methodology}

We performed a search of Pubmed, Embase and Cochrane using the following keywords: urachus cancer, urachus carcinoma, carcinoma of the urachus, cancer of the urachus, urachal cancer.

Guidelines from the European Association of Urology (EAU), the National Comprehensive Cancer Network (NCCN) and provincial guidelines from the British Columbia Cancer Agency (BCCA) were reviewed. Only the BCCA guidelines mention urachal cancer.

The first draft was written and reviewed by the project leaders (ZH and NB) and disseminated to GUMOC members for a primary review. The updated version was resubmitted to the group as well as key canadian representatives in the fields of urologic oncology, radiation oncology, pathology, and to a patient advocate. Consensus was obtained within the group by the revision of the summary statements until a unanimous agreement was achieved (either by email exchanges or in person discussions at the annual GUMOC meeting).

Guidelines for recommendations are described using the World Health Organisation (WHO) modified Oxford Centre for Evidence-based Medicine grading system. The level of evidence was described according to the following: Level 1: systematic review of randomized controlled trials (RCT); Level 2: individual RCT, including low-quality RCT; Level 3: controlled cohort; Level 4: case-control studies or case series; Level 5: expert opinion, mechanism-based reasoning. ${ }^{2}$

\section{Data source}

Since 1960, approximately 300 publications are available. Because of the scarcity of this entity, there are no prospective or phase III studies. For the purpose of this article, we included reviews and case series of 10 or more individuals with urachal cancer, with the exception of articles regarding molecular biology and emerging therapies that included fewer patients. Interestingly, the last two years have seen the publication of two important informative papers. First, the largest population-based study using the National Cancer Institute's Surveillance, Epidemiology, and End Results (SEER) database was published. This study comprises data from 18 cancer registries based on the 2010 census of the US population and contained 420 patients with urachal cancers. ${ }^{3}$ Secondly, a metaanalysis including most of the already described and published case series with a total of 1010 patients is now available. ${ }^{4}$

\section{Anatomy}

The urachus is a fibrous remnant of the allantois, a canal located at the bladder apex that drains the urinary bladder of the fetus. ${ }^{5}$ It extends upward from the anterior dome of the 
bladder toward the umbilicus and measures 3 to $10 \mathrm{~cm}$ in length and 8 to $10 \mathrm{~mm}$ in diameter. ${ }^{6}$ The urachus normally regresses during fetal life but the lumen of the urachus persists in approximately one-third of adults and traverses the bladder wall as a midline tubular or cystic structure of little clinical significance. ${ }^{78}$ The urachus comprises three different tissue layers: an epithelial canal lined by urothelium, submucosal connective tissues and an outer layer of smooth muscle. Urachal neoplasms can arise in any of these layers, and can be epithelial or mesenchymal. Moreover, the epithelium often demonstrates focal glandular metaplasia, providing a morphologic basis for the development of intestinal-type tumors. ${ }^{1}$

\section{Epidemiology}

The predominant histological type of bladder cancer in North America and Europe is urothelial (transitional cell) carcinoma in over $90 \%$ of cases. Adenocarcinoma of the bladder represents only $1.4 \%$. Among these rare cases of adenocarcinoma of the bladder, urachal cancer represents $10-40 \%$. The estimated annual incidence of this tumor in the general population is reported to be 1 in 5 million individuals. ${ }^{9}$ It is important to differentiate urachal adenocarcinoma from non-urachal adenocarcinoma of the bladder as the surgical approach is different for these two entities (extended partial cystectomy opposed to radical cystectomy for the latter). Furthermore, although the biology of adenocarcinoma of the urachus vs bladder is perceived to be similar, this has not been adequately studied in the literature.

Patients with urachal cancer are more likely to be male with a male to female ratio of 1.44:1 in the SEER database and 60\% males in the meta-analysis. ${ }^{34}$ Based on 17 reports, the median age at diagnosis is 52 years (range: 20-90 years) which is younger than non-urachal adenocarcinomas (median age 69). ${ }^{4-14}$

In the largest series, 5-year overall survival is about 50\%. ${ }^{9101213}$ The poor prognosis is mainly explained by advanced stage at presentation due to the relative lack of symptoms in localized disease, , difficulty of identification through cystoscopy and the absence of clear consensus in the investigation and treatment of localized and advanced disease. ${ }^{5}$ Despite a younger age of presentation and a prognosis as stated above, urachal cancers are associated with a superior survival compared to non-urachal adenocarcinomas. ${ }^{13} 15$

\section{Histology}

Adenocarcinoma is the most common histological subtype of urachal cancer representing $90 \%$ and $82.4 \%$ of the cases in the Mayo clinic series (420 patients) and in the SEER database respectively. ${ }^{310}$ Adenocarcinoma subtypes include mucinous adenocarcinoma, reported most frequently ${ }^{12}$, adenocarcinoma not otherwise specified and adenocarcinoma with signet ring cell morphology. Other histological subtypes are relatively uncommon 
and include squamous cell carcinoma, urothelial carcinoma and sarcoma. ${ }^{710}$ More than one histological subtype can be present in the same patient. ${ }^{12}$ Overall survival seems to be different for the different histologies of urachal cancer favouring the urothelial cell subtype. In the SEER database, urothelial cell carcinomas subtype had lower cancerspecific mortality than those with signet-cell tumors (HR $=6.14$; 95\% CI: 2.14-17.6; $\mathrm{p}<$ 0.001), adenocarcinoma NOS (HR = 3.09; 95\% CI: 1.21-7.84; $\mathrm{p}=0.001)$, and mucinous adenocarcinoma subtypes (HR $=3.01 ; 95 \% \mathrm{CI}: 1.21-7.5$; $\mathrm{p}=0.01)$.

\section{Immunohistopathology}

There are no specific markers to confirm or disprove urachal cancer. Moreover, there is a resemblance of these cells to gastric and colonic mucosa which may confuse the diagnosis. Indeed, a theory holds that progenitor cells for urachal tumors arise from enteric remnants left behind during embryologic development.

First, significant overlap seems to be present between adenocarcinomas of enteric, ovarian and urachal origin in regard to cytokeratin 7 (CK7), cytokeratin 20 (CK20) and CDX2 staining. However, CK7 tends to be negative in colonic or gastric signet cell cancers to the contrary of urachal cancer which is typically CK7 positive. ${ }^{16}$

Other potential markers include 34BE12 and beta catenin. Gopalan et al. performed immunohistochemistry (IHC) using CDX2, CK7, CK20, beta-catenin and 34BE12 on 15 adenocarcinomas of the urachus and compared them to 81 colonic adenocarcinomas. All cases stained positive for cytokeratin 20 and CDX2 while half of them stained positive for cytokeratin 7 . Approximately $2 / 3$ of patient tumors stained with 34BE12, and in the majority of these, only focal staining was seen. More than $90 \%$ of the urachal tumors showed strong and diffuse cytoplasmic membranous reactivity for beta catenin without nuclear staining. In comparison, 25\% of colonic adenocarcinoma showed cytoplasmic membranous reactivity with focal or diffuse nuclear accentuation and only $11 \%$ showed rare or focal cytoplasmic reactivity with 34BE12. The authors conclude in this review that immunostains do not unequivocally discriminate a urachal from a colorectal carcinoma, but diffuse positivity for 34BE12 would support a diagnosis of urachal carcinoma while a diffuse nuclear immunoreactivity for beta-catenin would militate against it. ${ }^{17}$

\section{Molecular biology}

Until 2016, little to no data was accessible in the medical literature regarding the biology of this particular tumor. In 2016, Modos et al. reported on mutational hot-spots, specifically KRAS, NRAS, BRAF, EGFR and PIK3CA genes in 22 urachal carcinoma samples. ${ }^{18}$ These five genes were selected because of the similarity between urachal carcinoma and colorectal adenocarcinoma. They found 11 mutations in 10 of 22 patients. KRAS was the most frequently affected (27\%), followed by BRAF (18\%) and NRAS 
(5\%). Unfortunately, no correlation was found between mutational status and clinicopathologic characteristics including signet ring cell differentiation, presence of calcification, clinical stage, tumor grade and the presence of lymph nodes or distant metastases and survival. Of interest, KRAS mutations were only present in nonmetastatic cases. Other smaller case series looked at mutational status of KRAS in urachal carcinoma. Sirintrapun et al. observed that 3 of 7 cases presented with KRAS mutation while Alexander et al. found a mutation in 1 of 5 cases. ${ }^{19}{ }^{20}$ On the basis of these small series, KRAS mutations may be more frequent in urachal carcinoma, similar to colorectal carcinoma, in contrast to urothelial carcinoma where they are not commonly described. Similarly, BRAF mutations are rare in urothelial carcinoma and adenocarcinoma of the bladder, but are present in $18 \%$ of urachal carcinoma the series described by Modos et al. ${ }^{18}$

\section{Clinical manifestations}

Macroscopic or microscopic hematuria is a frequent clinical presentation in about $80 \%$ of patients and implies that the tumor has transgressed the muscularis mucosae and invaded the urothelial surface. ${ }^{10}$ Other less frequent symptoms at presentation include bacteriuria, mucinuria, pain, abdominal mass and umbilicus infection. ${ }^{512}$ The median tumor size based on the largest diameter is reported to be between 3.0 and $6.3 \mathrm{~cm}$. Unfortunately, since the ligament lies outside the bladder, many patients present with locally advanced disease. For example, in the series of 151 urachal and

1374 nonurachal adenocarcinomas of the bladder patients by Wright et al., only $20 \%$ of the patients presented with localized disease. ${ }^{13}$ The tumor can extend toward nearby organs and metastases occur mainly in the pelvic lymph nodes, retroperitoneal lymph nodes, lungs and bones. There are no accurate statistics as to the frequency of metastatic sites, however, in a series by Ashley with 66 patients, systemic metastasis occurred in 39 patients at some point in the evolution of the disease (13 patients at diagnosis and 26 during follow up). Liver, pulmonary and bone were the most common. ${ }^{10}$

\section{Diagnostic modalities}

Different modalities used to establish the diagnosis include cytology, imaging, and cystoscopy. Cystoscopy can identify a visible mass in about $80 \%$ of patients whereas urine cytology will be positive in only $38 \%{ }^{10}{ }^{12}$. Urine cytology may be negative because of the extra vesical location of the tumor.

Radiographic imaging with CT scan provide strong supportive information of the diagnosis. On CT scan, urachal carcinoma may appear solid, cystic, or a combination of the two. Low-attenuation components are seen in $60 \%$ of cases, reflecting mucin content. Additionally, calcification within the tumor occurs in 50\%-70\% of cases and may be punctate, stippled, or curvilinear and peripheral. ${ }^{6}$ Therefore, the presence of a midline 
mass on the bladder dome, whether solid or cystic, especially with small calcifications, is considered highly suspicious, if not pathognomonic, for urachal cancer. ${ }^{21}$

With magnetic resonance imaging (MRI), the tumor is reported to be inhomogeneous and to show high intensity on T2-weighted images. Above all, MRI has the advantage of multiplane imaging and may be more useful than CT to determine the involvement of adjacent organs such as the bladder. In conclusion, both CT and MRI may be used in the clinical evaluation, to clarify the diagnosis for the former and evaluate locoregional extension for the latter. ${ }^{22}$

Finally, elevations of biomarkers such as CEA, CA-125, CA 19-9, may be elevated in $40-60 \%$ of patients; however, they are nonspecific and can be elevated in cancers of other origins. The response in these markers frequently correlate with radiographic response. ${ }^{21}$

\section{Staging}

Currently, there is no validated staging system for urachal cancer. Different staging approaches have been described, namely Sheldon, Mayo and modified TNM staging systems. The first two are the most commonly used.

Sheldon first described a staging system in 1984 (table1) and it remains the most reported classification, although was never officially validated. Ashley et al. from the Mayo Clinic described a second system based on 66 patients (table 2). They found the Sheldon staging system to be more complex and over-specified with its eight categories, 4 of which were occupied by $\leq 2$ patients, ${ }^{10}$ while the Mayo system was found to be more balanced in terms of patient distribution. Regardless, both systems predicted cancerspecific mortality equally well as shown in Table $3 .^{10}$

In a more recent publication, a second team from the Mayo Clinic chose to adapt the more universal TNM staging system to urachal cancer (table 4).

- GUMOC statement: The group favors the use of the modified TNM staging system. However, all three staging system give relevant information of prognostic utility and should be obtained for every patient, when feasible (Level of evidence 4, Grade C).

\section{Diagnosis}

\section{Diagnostic criteria}

The criteria for diagnosis of urachal carcinoma most agreed upon are those described by Sheldon et $\mathrm{al}^{23}$ and Mostofi et $\mathrm{al}^{24}$. These include (a) tumor in the dome of the bladder; (b) absence of cystitis cystica and cystitis glandularis; (c) predominant invasion of the muscularis or deeper tissues with a sharp demarcation between the tumor and surface bladder urothelium which is free of glandular or polypoid proliferation; (d) presence of 
urachal remnants within the tumor; (e) extension of tumor into the bladder wall with involvement of the space of Retzius, anterior abdominal wall or umbilicus, and (f) no evidence of a primary neoplasm elsewhere. ${ }^{17}$ In 2016, Paner et al. published an update in the pathologic diagnosis and classification of epithelial neoplasms of urachal origin. ${ }^{15}$ They found the original criteria above too restrictive and proposed modified criteria. Indeed, urachal remnants were reported in only $15 \%$ to $62.5 \%$ of cases. ${ }^{17}$ Cystitis cystica and cystitis glandularis were identified focally in $17 \%$ to $21 \%$ of cases and while the majority of urachal tumors were situated at the dome, $4 \%$ of the cases could be in the anterior wall. ${ }^{17} 25$

- Based on proposals from Paner et. al, GUMOC supports the use of the following modified criteria: (1) location of the tumor in the dome or anterior wall; (2) epicenter of the tumor in the bladder wall; (3) absence of widespread adenomatous changes and widespread cystitis cystica/ cystitis glandularis in the bladder wall; (4) absence of urothelial neoplasia in the bladder and (5) absence of a known primary elsewhere.

\section{Prognostic factors}

The five prognostic factors more frequently described and most validated include: stage of the disease, presence of positive margins after surgery, pathological tumor grade, presence of positive lymph nodes and type of surgery. The latter refers to complete en bloc resection of the urachus and the umbilical cord versus an incomplete resection. Several studies have shown that the presence of an advanced stage is associated with a poor prognosis. ${ }^{1314}$. Using the TNM staging system reported above, Molina and al. from Mayo Clinic showed that median survival is more than 10 years for stage I and 7.5 years for stage II. It then decreases significantly to 1-2 years and less than one year for stages III and IV, respectively. 12

Second, the ability to achieve negative margins has a strong impact on survival. In the study by Ashley et al, presence of positive margins was associated with a hazard ratio of cancer-specific mortality of $3.8(\mathrm{p}<0,001){ }^{10}$

Third, tumor grade remains one of the most important prognostic factors in multivariate analysis. In the study by Pinthus et al., patients with a well-differentiated operated primary tumor had up to $90 \%$ long-term disease specific survival, compared to patients with poorly differentiated tumors who all died. ${ }^{9}$

Fourth, the additional finding of lymph node metastasis, occult or not, has been associated with adverse prognosis on multivariate analysis in different series. Again, in the study by Ashley and al., presence of lymph node metastasis was associated with a hazard ratio of cancer-specific mortality of 5.1 ( $\mathrm{p}<0.001){ }^{10}$

- GUMOC statement: The 5 main prognostic factors in urachal cancers 


\section{that should be evaluated for every patient include: stage of the disease, surgical margin status, pathological tumor grade, presence of positive lymph nodes and type of surgery (Level of evidence 4, Grade C).}

\section{Treatment}

\section{Surgery}

The recommended treatment for localized urachal cancer is en bloc surgical removal of the umbilicus with the urachal ligament and partial cystectomy. This intervention is associated with the highest median survival when compared to cystectomy without

umbilectomy. ${ }^{12627}$ Herr et al. reported the survival of 50 patients operated in their institution for urachal carcinoma. They found that en bloc resection of the urachal tumor and urachus combined with partial cystectomy cured $70 \%$ of the patients with clinically localized urachal cancer. ${ }^{11}$ In another series, although en bloc resection was not statistically associated with survival ( $p=0.09), 13$ of the 16 long-term survivors after resection were in the group treated with en bloc resection and umbilectomy. ${ }^{26}$

The large meta-analysis included the surgical treatment for 957 patients ${ }^{4}: 66 \%$ had partial cystectomy while $12 \%$ underwent radical cystectomy. In $67 \%$ of the patients, umbilectomy was performed. These data suggest that en bloc surgical removal of the umbilicus with the urachal ligament concurrently to partial cystectomy is commonly performed and may be associated with better outcome based on retrospective series. ${ }^{4}$ As for extensive pelvic lymphadenectomy, it does not seem to improve survival over that of the aforementioned surgery and seems to be associated with more complications as reported in the majority of the literature. ${ }^{14}$ Only $38 \%$ patients in the meta-analysis had lymphadenectomy and $17 \%$ of these cases had positive lymph nodes. In the SEER database, lymphadenectomy seems to be associated with radical cystectomy as $8 \%$, $44.1 \%$ and $78 \%$ of patients who underwent local excision, partial and radical cystectomy respectively had documented lymph nodes removal. In total, only $13.4 \%$ had positive lymph nodes. ${ }^{3}$ Although pelvic lymphadenectomy is advocated in patients with urachal cancers, its therapeutic role as well as the template of dissection needs to be further defined and studied.

Finally, there is no apparent survival advantage associated with complete cystectomy versus partial cystectomy. ${ }^{27}$ According to Siefker et al., complete cystectomy with en bloc resection of the urachal ligament and umbilicus should be considered only if necessary to obtain negative margins or if partial cystectomy would result in inadequate bladder capacity and function ${ }^{21}$.

\section{- GUMOC statements:}

- En bloc surgical removal of the umbilicus, urachal ligament, and partial cystectomy with pelvic lymphadenectomy is the preferred 
intervention. Radical cystectomy with en bloc resection of the urachal ligament and umbilicus should be considered in selected cases when negative bladder surgical margins is not possible with a partial cystectomy (Level of evidence 4, Grade C).

- In patients with a positive margin and/or incomplete surgical procedure, consideration should be given to second look surgery (Level of evidence 5, expert opinion).

- In the case of oligometastatic disease or localised recurrence, surgical resection can be discussed on a case-by-case basis (Level of evidence 5, expert opinion).

\section{Radiotherapy}

Urachal tumors are not particularly radiosensitive and radiotherapy is rarely used. In the SEER database, only $10 \%$ of patients (total 414 patients) received radiotherapy, 29 in combination with surgery (13 stage IV, 10 stage III and 5 stage II disease) and 13 as monotherapy (of which 11 were stage IV). ${ }^{3}$ It is sometimes used post-operatively for positive margins or for localized inoperable disease, but without any strong evidence to suggest improved curability ${ }^{21}$.

- GUMOC statement: Surgery should be the preferred modality in localized disease. When patients are considered unfit for surgery, radiotherapy can be considered, but the benefits are unclear. Radiation therapy can be considered for post-operative positive margins especially if second look surgery is not deemed feasible. Palliative radiation can be considered for incurable disease (Level of evidence 5, Expert opinion).

\section{Chemotherapy}

No recommendations exist with respect to the use and type of chemotherapy in the perioperative setting (neoadjuvant or adjuvant).

However, there are some case reports of patients who have received post-operative chemotherapy due to positive margins, lymph node or peritoneal involvement, or unresected umbilicus and a high likelihood of relapse ${ }^{21}$.

In advanced disease, it is difficult to draw any conclusions regarding the best chemotherapy regimen from published series, as types of treatment used are heterogeneous and the number of patients small. There is certainly a medical need to determine the optimal and more effective treatments as 5-year overall survival for metastatic disease is less than $20 \%$. Despite this, the main drug used is cisplatin alone or in combination such as cisplatin-methotrexate-vinblastine (CMV), methotrexatevinblastine-doxorubicin-cyclophosphamide (MVAC), cisplatin-5-FU-gemcitabine or others. Additional reported drugs include paclitaxel, cisplatin and ifosfamide. ${ }^{52128}$ 
Because of a certain resemblance to colonic cancer in terms of immunohistochemistry, elevation of CEA and production of mucin by the tumor, regimens described in recent published case reports are derived from those often offered in gastrointestinal cancers FOLFOX and FOLFIRI. ${ }^{29} 30$

In the large meta-analysis, the authors compared the efficacy of cisplatin-based, 5FU-based, 5-FU+cisplatin combination and other chemotherapies. A total of 74 patients were analyzed. The response rate was higher in the 5-FU and the 5-FU+cisplatin groups (44\% and 43\% respectively) in contrast to the non 5-FU group having a lower response rate (9\%). The combination of 5-FU+cisplatin had also the lowest progression rate and thus seems to be have the best outcomes in this report. In this paper, regimens using these two drugs included: 5-FU+leucovorin, gemcitabine, cisplatin; 5-FU+IFN-a, cisplatin; 5FU+oxaliplatin; and 5-FU+cisplatin. ${ }^{4}$

Other case series are available and presented in table 5. They all include a smaller number of patients due to this diagnosis rarity. Hong et al recently reported a retrospective study of 21 patients with non-transitional cell bladder carcinoma including 4 urachal cancer patients. The overall response rate was 33\% and the median survival 13 months. Within the study, 11 patients received gemcitabine-cisplatin, 6 5-FU-cisplatin, 1 Paclitaxel-cisplatin, 1 MVAC, 1 CMV and 1 VIP (etoposide-ifosfamide-cisplatin). ${ }^{31}$ In another prospective study by Galsky et al., 20 patients including 6 urachal cancer patients received ifosfamide, paclitaxel and cisplatin. Overall, 35\% achieved major response and the median survival time of patients with adenocarcinoma was 24.8 months. $^{32}$

Finally, another major series by Siefker et al. reported a 33\% overall response rate when using a platinum based regimen. Of the 20 patients who received chemotherapy, four partial or complete responses were observed, including three following a protocol based on 5-FU and cisplatin. $^{26}$

The latest publication is by Jung et al. and demonstrates the heterogeneity of treatments with 10 urachal cancer patients receiving a total of 24 different schemes of palliative chemotherapy. Regimens with a base of 5-FU, taxane and gemcitabine were the most common and the overall response rate of all chemotherapeutic regimens was $16.7 \%{ }^{28}$

\section{Targeted therapy}

Because of the similarity previously described between urachal and colorectal cancer, the use of epidermal growth factor receptor (EGFR)-inhibitors as in colorectal cancer would theoretically be feasible. On this subject, very little but nonetheless interesting data is available. In a phase I study of gefitinib published in 2005, one patient with urachal cancer was included and had a transient $55 \%$ decrease in tumor size. ${ }^{33}$ Recently, Collazo-Lorduy et al. published a report with a patient with metastatic urachal cancer in whom targeted exome sequencing revealed EGFR amplification and wild-type KRAS. ${ }^{34}$ 
The patient was treated with cetuximab as a single agent providing him with a response lasting more than 8 months. Subsequently, targeted exome sequencing was performed on nine other specimens finding Mitogen-activated-protein-kinase (MAPK) pathway mutations in 4 out of 9 cases but no EGFR amplification. Moreover, 2 cases harbored APC mutations. To conclude, EGFR-inhibitor therapy is certainly of interest in these cases, however further evaluations of the genetic features of this disease are warranted and may lead to more rational targeted approaches in the future.

\section{Immunotherapy}

Immunotherapy changed the paradigm of treatment of many cancers in the last few years. Unfortunately, to our knowledge, there are no cases of urachal cancer treated with checkpoint inhibitors that have been published so far. However, it was recently shown that mismatch-repair status predicted clinical benefit of immune checkpoint blockade with anti-PD1 therapy. ${ }^{35}$ Interestingly, Sirintrapun et al. evaluated microsatellite instability (MSI) in 7 cases with urachal cancer. ${ }^{19}$ Three cases showed MSI, 1 with MSH2 and MSH6 and 2 with PMS2 loss. Further evaluation of a larger cohort is certainly desirable to explore possibility of treatment with immune compounds.

- GUMOC statements:

- The benefit of adjuvant chemotherapy is unknown at this time and is not recommended on a routine basis. The optimal regimen in this setting has not been defined

- In the absence of high level evidence, we suggest FOLFOX as the preferred regimen. The chemotherapy regimen can be tailored to immunohistopathological findings, especially if urothelial carcinoma features on histology is present (Level of evidence 4, Grade C).

- Given the rarity of this tumor, patients with this diagnosis should be discussed at a multidisciplinary forum and every effort should be made for the patient to be seen or discussed in collaboration with specialists who have some experience with this cancer. Ideally, a molecular profile should be obtained to identify potentially biological pathways and actionable targets to guide treatment. Finally, participation in clinical trials where possible is encouraged.

\section{Conclusions}

Urachal cancer is a rare entity with a poor prognosis when presenting with advanced stage. Collaborative efforts are warranted in order to improve patient outcomes and 
formalize treatment modalities. Examples include the establishment of a national or international clinical database and standardization of treatment approaches in reference cancer institutions. Better knowledge of this tumor's molecular features may also improve pathological definition and define targeted approaches for this advanced disease. 


\section{Hamilou et al Management of urachal cancer}

\section{References}

1. Begg RC. The Urachus: its Anatomy, Histology and Development. J Anat 1930;64(Pt 2):170-83. [published Online First: 1930/01/01]

2. Bob Phillips CB, Dave Sackett et. al Levels of Evidence 2009 University of Oxford, Graduate Schoolin EBM and Research Methods, Centre for Evidence-based Medicine 1998 [updated March 2009. Available from: http://www.cebm.net/index.aspx?0=1025 accessed January 2019.

3. Mylonas KS, P OM, Ziogas IA, et al. Malignant urachal neoplasms: A population-based study and systematic review of literature. Urol Oncol 2017;35(1):33 e11-33 e19. doi: 10.1016/j.urolonc.2016.07.021

4. Szarvas T, Modos O, Niedworok C, et al. Clinical, prognostic, and therapeutic aspects of urachal carcinoma-A comprehensive review with meta-analysis of 1,010 cases. Urol Oncol 2016;34(9):388-98. doi: 10.1016/j.urolonc.2016.04.012

5. Peugniez C, Ghoneim T, Leroy X, et al. [Urachal cancers]. Bull Cancer 2013;100(5):50917. doi: 10.1684/bdc.2013.1718 [published Online First: 2013/03/19]

6. Yu JS, Kim KW, Lee HJ, et al. Urachal remnant diseases: spectrum of CT and US findings. Radiographics 2001;21(2):451-61. doi:

10.1148/radiographics.21.2.g01mr02451 [published Online First: 2001/03/22]

7. Zhang J, Wu J. Options for diagnosis and treatment of urachal carcinoma. Asia Pac $J$ Clin Oncol 2013;9(2):117-22. doi: 10.1111/j.1743-7563.2012.01592.x [published Online First: 2012/10/11]

8. Beck AD, Gaudin HJ, Bonham DG. Carcinoma of the urachus. Br J Urol 1970;42(5):555-62. [published Online First: 1970/10/01]

9. Pinthus JH, Haddad R, Trachtenberg J, et al. Population based survival data on urachal tumors. J Urol 2006;175(6):2042-7; discussion 47. doi: 10.1016/S0022-5347(06)00263-1 [published Online First: 2006/05/16]

10. Ashley RA, Inman BA, Sebo TJ, et al. Urachal carcinoma: clinicopathologic features and long-term outcomes of an aggressive malignancy. Cancer 2006;107(4):712-20. doi: 10.1002/cncr.22060 [published Online First: 2006/07/11]

11. Herr HW, Bochner BH, Sharp D, et al. Urachal carcinoma: contemporary surgical outcomes. J Urol 2007;178(1):74-8; discussion 78. doi: 10.1016/j.juro.2007.03.022 [published Online First: 2007/05/15]

12. Molina JR, Quevedo JF, Furth AF, et al. Predictors of survival from urachal cancer: a Mayo Clinic study of 49 cases. Cancer 2007;110(11):2434-40. doi: 10.1002/cncr.23070 [published Online First: 2007/10/13] 


\section{Hamilou et al Management of urachal cancer}

13. Wright JL, Porter MP, Li CI, et al. Differences in survival among patients with urachal and nonurachal adenocarcinomas of the bladder. Cancer 2006;107(4):721-8. doi: 10.1002/cncr.22059 [published Online First: 2006/07/11]

14. Bruins HM, Visser O, Ploeg M, et al. The clinical epidemiology of urachal carcinoma: results of a large, population based study. J Urol 2012;188(4):1102-7. doi: 10.1016/j.juro.2012.06.020 [published Online First: 2012/08/21]

15. Paner GP, Lopez-Beltran A, Sirohi D, et al. Updates in the Pathologic Diagnosis and Classification of Epithelial Neoplasms of Urachal Origin. Adv Anat Pathol 2016;23(2):71-83. doi: 10.1097/PAP.0000000000000110

16. Behrendt MA, van Rhijn BW. Genetics and biological markers in urachal cancer. Transl Androl Urol 2016;5(5):655-61. doi: 10.21037/tau.2016.04.01

17. Gopalan A, Sharp DS, Fine SW, et al. Urachal carcinoma: a clinicopathologic analysis of 24 cases with outcome correlation. Am J Surg Pathol 2009;33(5):659-68. doi:

10.1097/PAS.0b013e31819aa4ae [published Online First: 2009/03/03]

18. Modos O, Reis H, Niedworok C, et al. Mutations of KRAS, NRAS, BRAF, EGFR, and PIK3CA genes in urachal carcinoma: Occurence and prognostic significance. Oncotarget 2016;7(26):39293-301. doi: 10.18632/oncotarget.9828

19. Sirintrapun SJ, Ward M, Woo J, et al. High-stage urachal adenocarcinoma can be associated with microsatellite instability and KRAS mutations. Hum Pathol 2014;45(2):327-30. doi: 10.1016/j.humpath.2013.09.008

20. Alexander RE, Lopez-Beltran A, Montironi R, et al. KRAS mutation is present in a small subset of primary urinary bladder adenocarcinomas. Histopathology 2012;61(6):1036-42. doi: 10.1111/j.1365-2559.2012.04309.x

21. Siefker-Radtke A. Urachal adenocarcinoma: a clinician's guide for treatment. Semin Oncol 2012;39(5):619-24. doi: 10.1053/j.seminoncol.2012.08.011 [published Online First: 2012/10/09]

22. Krysiewicz S. Diagnosis of urachal carcinoma by computed tomography and magnetic resonance imaging. Clin Imaging 1990;14(3):251-4. [published Online First: 1990/08/01]

23. Sheldon CA, Clayman RV, Gonzalez R, et al. Malignant urachal lesions. J Urol 1984;131(1):1-8. [published Online First: 1984/01/01]

24. Mostofi FK, Thomson RV, Dean AL, Jr. Mucous adenocarcinoma of the urinary bladder. Cancer 1955;8(4):741-58. [published Online First: 1955/07/01]

25. Dhillon J, Liang Y, Kamat AM, et al. Urachal carcinoma: a pathologic and clinical study of 46 cases. Hum Pathol 2015;46(12):1808-14. doi: 10.1016/j.humpath.2015.07.021

26. Siefker-Radtke AO, Gee J, Shen Y, et al. Multimodality management of urachal carcinoma: the M. D. Anderson Cancer Center experience. J Urol 2003;169(4):1295-8. doi: 10.1097/01.ju.0000054646.49381.01 [published Online First: 2003/03/12] 


\section{Hamilou et al Management of urachal cancer}

27. Henly DR, Farrow GM, Zincke H. Urachal cancer: role of conservative surgery. Urology 1993;42(6):635-9. [published Online First: 1993/12/01]

28. Jung HA, Sun JM, Park SH, et al. Treatment outcome and relevance of palliative chemotherapy in urachal cancer. Chemotherapy 2014;60(2):73-80. doi:

10.1159/000368071 [published Online First: 2014/12/05]

29. Kikuchi M, Kamei S, Morirama Y, et al. [Case of urachal cancer treated by neoadjuvant chemotherapy with FOLFOX4 (oxaliplatin, 5-FU and leukovolin)]. Hinyokika Kiyo 2008;54(8):557-9. [published Online First: 2008/09/16]

30. Kume H, Tomita K, Takahashi S, et al. Irinotecan as a new agent for urachal cancer. Urol Int 2006;76(3):281-2. doi: 10.1159/000091635 [published Online First: 2006/04/08]

31. Hong JY, Choi MK, Uhm JE, et al. Palliative chemotherapy for non-transitional cell carcinomas of the urothelial tract. Med Oncol 2009;26(2):186-92. doi: 10.1007/s12032008-9106-7 [published Online First: 2008/11/07]

32. Galsky MD, Iasonos A, Mironov S, et al. Prospective trial of ifosfamide, paclitaxel, and cisplatin in patients with advanced non-transitional cell carcinoma of the urothelial tract. Urology 2007;69(2):255-9. doi: 10.1016/j.urology.2006.10.029 [published Online First: 2007/02/27]

33. Goss G, Hirte H, Miller WH, Jr., et al. A phase I study of oral ZD 1839 given daily in patients with solid tumors: IND.122, a study of the Investigational New Drug Program of the National Cancer Institute of Canada Clinical Trials Group. Invest New Drugs 2005;23(2):147-55. doi: 10.1007/s10637-005-5860-y

34. Collazo-Lorduy A, Castillo-Martin M, Wang L, et al. Urachal Carcinoma Shares Genomic Alterations with Colorectal Carcinoma and May Respond to Epidermal Growth Factor Inhibition. Eur Urol 2016;70(5):771-75. doi: 10.1016/j.eururo.2016.04.037

35. Le DT, Uram JN, Wang H, et al. PD-1 Blockade in Tumors with Mismatch-Repair Deficiency. N Engl J Med 2015;372(26):2509-20. doi: 10.1056/NEJMoa1500596 
Figures and Tables

\begin{tabular}{|l|r|l|}
\hline \multicolumn{2}{|l|}{ Table 1. Sheldon staging system of urachal cancer } \\
\hline Stage & \multicolumn{2}{|c|}{ Sheldon staging system } \\
\hline I & & Urachal cancer confined to urachal mucosa \\
\hline II & & Urachal cancer with invasion confined to urachal itself \\
\hline III & A & Local urachal cancer extension to bladder \\
\hline & B & Local urachal cancer extension to abdominal wall \\
\hline & C & Local urachal cancer extension to peritoneum \\
\hline & D & Local urachal cancer extension to viscera other than bladder \\
\hline IV & A & Metastatic urachal cancer to lymph nodes \\
\hline & B & Metastatic urachal cancer to distant sites \\
\hline
\end{tabular}

\begin{tabular}{|l|l|}
\hline \multicolumn{2}{|l|}{ Table 2. Mayo staging system of urachal cancer } \\
\hline Stage & \\
\hline I & Tumor confined to urachus and/or bladder \\
\hline II & $\begin{array}{l}\text { Tumor extending beyond the muscular layer of } \\
\text { urachus and/or bladder }\end{array}$ \\
\hline III & Tumor infiltrating the regional lymph node \\
\hline IV & $\begin{array}{l}\text { Tumor infiltrating non-regional lymph nodes or } \\
\text { distant sites }\end{array}$ \\
\hline
\end{tabular}


Table 3. Cancer-specific survival in patients with urachal carcinoma as per Sheldon and Mayo clinical staging system

\begin{tabular}{|l|c|c|}
\hline Stage & 5-year cancer-specific survival & 10-year cancer-specific survival \\
\hline I & $63 \%$ & $57 \%$ \\
\hline II & $55 \%$ & $46 \%$ \\
\hline III & $19 \%$ & $0 \%$ \\
\hline IV & $8 \%$ & $0 \%$ \\
\hline
\end{tabular}




\begin{tabular}{|l|l|}
\hline \multicolumn{2}{|l|}{ Table 4. Modified TNM staging system of urachal cancer by Mayo clinic } \\
\hline T stage & \\
\hline Tis & $\begin{array}{l}\text { Tumor localized to the urachal mucosa with no invasion to the basal } \\
\text { membrane (carcinoma in situ) }\end{array}$ \\
\hline T1 & Tumor with invasion through the basal membrane \\
\hline T2a & Tumor invades deep muscle (outer half) \\
\hline T2b & Tumor invades the superficial muscle of the bladder (inner half) \\
\hline T3 & $\begin{array}{l}\text { Tumor invades perivesical fat, abdominal wall muscle (in cases of extravesi- } \\
\text { cal urachal tumors) }\end{array}$ \\
\hline N stage & \\
\hline NX & Lymph nodes cannot be assessed \\
\hline N0 & No lymph node metastasis \\
\hline N1 & $\begin{array}{l}\text { Single regional lymph node metastasis in the true pelvis (hypogastric, } \\
\text { obturator, external iliac, or presacral lymph node) }\end{array}$ \\
\hline N2 & $\begin{array}{l}\text { Multiple regional lymph node metastasis in the true pelvis (hypogastric, } \\
\text { obturator, external iliac, or presacral lymph node metastasis) }\end{array}$ \\
\hline N3 & Lymph node metastasis to the common iliac lymph nodes \\
\hline M stage & \\
\hline M0 & No distant metastasis \\
\hline M1 & Distant metastasis \\
\hline
\end{tabular}




\begin{tabular}{|c|c|c|c|c|c|}
\hline Authors & N (total) & $\begin{array}{c}\text { Number of } \\
\text { urachus } \\
\text { adenocarcino } \\
\text { ma only }\end{array}$ & Regimens & Response rate $(\%)$ & $\begin{array}{l}\text { Mean survival time } \\
\text { (months) }\end{array}$ \\
\hline $\begin{array}{l}\text { Jung et al } \\
(2014)^{28}\end{array}$ & 10 & 10 & $\begin{array}{l}24 \text { different regiments. Most } \\
\text { common: 5-Fu, gemcitabine and } \\
\text { taxane based }\end{array}$ & 16.7 & Unknown \\
\hline $\begin{array}{l}\text { Hong et al } \\
(2009)^{31}\end{array}$ & 21 & 5 & $\begin{array}{c}\mathrm{GP}^{\dagger} \text {; FP; TP; MVAC; CMV; VIP. } \\
\text { TC } ; \text { EP; BOMP; VI; paclitaxel }\end{array}$ & $29^{\S} / 9^{q}$ & 13 \\
\hline $\begin{array}{l}\text { Galsky et al } \\
(2007)^{32}\end{array}$ & 20 & 6 & Ifosfamide, paclitaxel, and cisplatin & 35 & $\begin{array}{l}24.8 \text { (adeno)/ } \\
8.9 \text { (no-adeno) }\end{array}$ \\
\hline $\begin{array}{l}\text { Molina et al } \\
(2007)^{12}\end{array}$ & 10 & 10 & $\begin{array}{l}\text { 5-FU, FP 5-FU, } \\
\text { lomustine + vincristine; TP; platinum } \\
\text { and etoposide, MVAC }\end{array}$ & 56 & 20.4 \\
\hline $\begin{array}{l}\text { Siefker-Radtke } \\
\text { et al }(2003)^{26}\end{array}$ & 20 & 20 & $\begin{array}{l}\text { Emory }^{\dagger \dagger}, \text { 5-FU, } \alpha \text {-interferon, } \\
\text { cisplatin, MVAC, paclitaxel-based }{ }^{\ddagger \ddagger} \text {, } \\
\text { ifosfamide-based }{ }^{\S \S}\end{array}$ & 33 & 20 \\
\hline
\end{tabular}

${ }^{\dagger}$ First-line chemotherapy regimen. ${ }^{\ddagger}$ Second-line or third-line chemotherapy regimen. ${ }^{\S}$ Response rate of first-line chemotherapy. "Response rate of second-line chemotherapy. ${ }^{\dagger \dagger} 5$-FU, leucovorin + gemcitabine + cisplatin. ${ }^{\sharp}$ Paclitaxel + 
carboplatin, paclitaxel + cisplatin, paclitaxel+ methotrexate + cisplatin, and paclitaxel alone. ${ }^{\S \S}$ Ifosfamide, cisplatin + gemcitabine, 5-FU, 5-fluorouracil. Adeno: adenocarcinoma; BOMP: bleomycin + vincristine + mitomycin + cisplatin; CMV: methotrexate + vinblastine + cisplatin; EP: etoposide + cisplatin; FP: 5-FU + cisplatin; GP: gemcitabine + cisplatin; MVAC: methotrexate + vinblastine + doxorubicin + cisplatin; no-adeno: non-adenocarcinoma; TC: paclitaxel + carboplatin; TP: paclitaxel + cisplatin; VI: VP-16 + ifosfamide; VIP: VP-16 + ifosfamide + cisplatin. 